\title{
$\mathrm{W}_{6} \mathrm{Mo}_{5} \mathrm{Cr}_{4} \mathrm{~V}_{2}$ 高速钢低温强化机理
}

程本培 李勇陈 莉

(中国科学院固体物理研究所, 合肥 230031)

\author{
任连治 李彩云 \\ (核工业部西南物理研究院, 成都 610041)
}

\section{关键词 $\mathrm{W}_{6} \mathrm{Mo}_{5} \mathrm{Cr}_{4} \mathrm{~V}_{2}$ 高速钢 低温强化处理 微观结构}

80 年代初, 已经有关于深冷处理可以使工具钢及某些不锈钢制品的耐磨性和使用寿命提 高几倍的报道. 后来, 美、前苏联、日等国已将这种新技术应用于刀具、模具、量具、齿轮、涡轮轴、 弹簧等工件的强化处理 ${ }^{[1,2]}$, 取得了很好的经济效益. 但是, 对低温强化的机理研究工作却开 展得很少, 限制了它的进一步发展. 对这种处理的必要性、可靠性也存在一定的争论. 近几 年, 国内一些单位也开展了深冷处理工艺的实验研究工作, 并通过十几个厂家对各种高速钢 刀具在处理前后的耐磨性、使用寿命等性能进行了大量的工艺对比试验. 证实通过合适的深 冷处理工艺制度, 可以使它们的耐磨性和使用寿命普遍提高 1 3 倍. 本文研究了导致低温 强化的机制, 这对于指导改进工艺规程, 扩大应用范围, 都有着重要的意义.

\section{1 实验方法}

实验样品是从 $\mathrm{W}_{6} \mathrm{Mo}_{5} \mathrm{Cr}_{4} \mathrm{~V}_{2}$ 高速钢实用刀具上直接切割下来的, 该样品已按标准工艺进行 过淬火和回火处理.

在进行深冷处理之前, 首先对样品进行了 $X$ 射线物相分析、马氏体点阵常数测定、碳化物 相的线形分析、金相观察、显微硬度测量和扫描电镜观测. 然后, 按实用刀具的深冷处理工艺 对样品进行处理. 处理后, 再在同一观察面上, 按同样测试条件, 重复处理前的各项测试工 作, 以便于进行分析对比.

利用 LAS200 型二次离子质谱仪进行碳分布和碳含量的分析是对 $\mathrm{W}_{18} \mathrm{Cr}_{4} \mathrm{~V}$ 高速工具钢进 行的. 同样进行了深冷处理前后的对比实验.

\section{2 实验结果和讨论}

\section{$2.1 \mathrm{X}$ 射线衍射分析}

$\mathrm{X}$ 射线物相分析的结果如图 1 所示. 可以看出, 该试样的主相为马氏体, 由于含碳量不是 很高, 所以各主峰都未能分裂. 奥氏体含量很少, 碳化物相主要由 $\mathrm{M}_{6} \mathrm{C}$ 型碳化物和六方 VC 组成, 另外还有少量的面心立方 VC 相. 深冷处理前后 $\mathrm{X}$ 射线衍射图基本相同, 没有增加新 的物相, 也没有看到某个物相明显减少. 曾有一种观点, 认为低温强化的重要原因之一是大 量残余奥氏体向回火马氏体的转变 ${ }^{[1]}$. 但是, 至少对于高速工具钢刀具, 这一分析结论是不够 确切的.

1994-08-16 收稿, 1995-01-05 收修改稿 
我们用内标法测量了样品深冷处理前后马氏 体点阵参数的变化. 利用经验公式 ${ }^{[3]}: c=a_{0}+\alpha \rho$, $a=a_{0}-\beta \rho$ 可以由点阵参数的变化估算马氏体中 含碳量 $\rho$ 的变化. 式中 $a_{0}$ 为 $\alpha \mathrm{Fe}$ 点阵参数, $\alpha=0.116, \beta=0.013$. 由于样品中马氏体含碳量不 很高, 主峰未能分裂, 可以将上两式简化为 $(a+c) / 2=a_{0}+1 / 2(\alpha-\beta) \rho$. 由平均点阵参数的变 化估算出深冷处理前后马氏体中含碳量分别为 $0.35 \%$ 和 $0.25 \%$ (由于未考虑合金元素的影响, 这 是一种粗䊁的估算), 即深冷处理后马氏体中含 碳量进一步减少了近 $30 \%$, 所以又有大量新的碳 化物从马氏体相中析出. 这种析出仅引起马氏体 点阵参数的微小变化和碳化物线形的某些展宽, 因此, 在图 1 的普通 X 射线扫描图中难以察觉.

用线形分析的方法分析了 $M_{6} \mathrm{C}$ 型碳化物和六 方 $\mathrm{VC}$ 深冷处理前后线形的变化,并估算出它们的

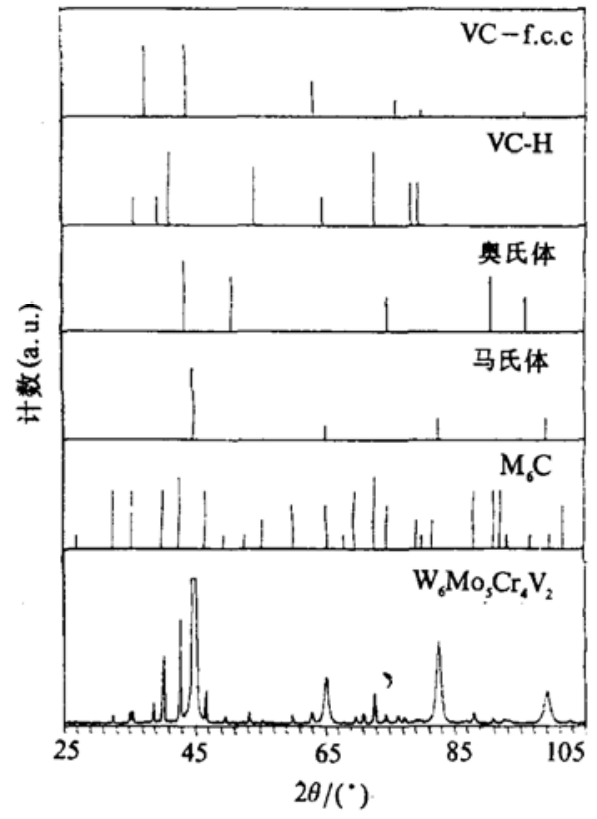

图 $1 \mathrm{X}$ 射线物相分析 平均晶粒粒度和平均相对应变. $\mathrm{M}_{6} \mathrm{C}$ 的实验结果示于表 1.

表 $1 \mathrm{M}_{6} \mathrm{C}$ 型碳化物的平均晶粒尺寸 $D$ 和平均相对应变 $e$

\begin{tabular}{cccc}
\hline 项目 & 深冷处理前 $/ \mathrm{nm}$ & 深冷处理后 $/ \mathrm{nm}$ & 冷后、冷前相对变化 $/ \%$ \\
\hline $\mathrm{D}$ & 201 & 181 & -11 \\
$\mathrm{e} / \%$ & 0.062 & 0.073 & +16 \\
\hline
\end{tabular}

由表 1 可看出, 深冷处理后 $\mathrm{M}_{6} \mathrm{C}$ 型 碳化物的平均晶粒尺寸减少了约 $11 \%$, 而相对应变增加了约 $16 \%$. 这是由于 深冷处理后析出的 $\mathrm{M}_{6} \mathrm{C}$ 型碳化物十分 细小和弥散, 致使平均晶粒尺寸减

小, 又由于它们是从晶粒内部析出的, 承受较大应力, 致使平均应变增加.

同样的测试方法应用于 VC 线形, 却未发现类似的变化. 这是由于 V 形成碳化物的倾向 最强 ${ }^{[4]}$, 淬火后就首先在晶界上析出不易熔解的, 十分稳定且细小 $($ 约 $0.1 \mu \mathrm{m})$ 的 VC 粒子. 回 火过程中又再次以细小质点弥散析出, 到深冷处理时已没有再析出 VC 的潜力. 所以处理前 后 $\mathrm{VC}$ 线形基本保持不变.

\section{2 显微硬度的测量}

样品深冷处理前后显微硬度 $\mathrm{HV}_{0.2}$ 的测量结果的统计平均值如表 2 所示.

表 2 深冷处理前后的显微硬度 $\mathrm{HV}_{0.2}$

\begin{tabular}{lcc}
\hline \multicolumn{1}{c}{ 测量位置 } & 深冷处理前 & 深冷处理后 \\
\hline 整体平均值 & 724 & 708 \\
大块碳化物分布区 & 781 & 745 \\
细小弥散碳化物分布区 & 668 & 687 \\
\hline
\end{tabular}
布弥散的碳化物小颗粒. 马氏体显微硬度值约为 HV 700随含 C 量不同而有所差异. 碳化物 HV 应大于 1000 . 由于高速钢中大的碳化物粒度也都小于压痕的尺寸, 因 此, 即使压痕打在大块碳化物上, 亦是碳化物与马氏体的综合作用结果, HV 值仅稍高于马氏 


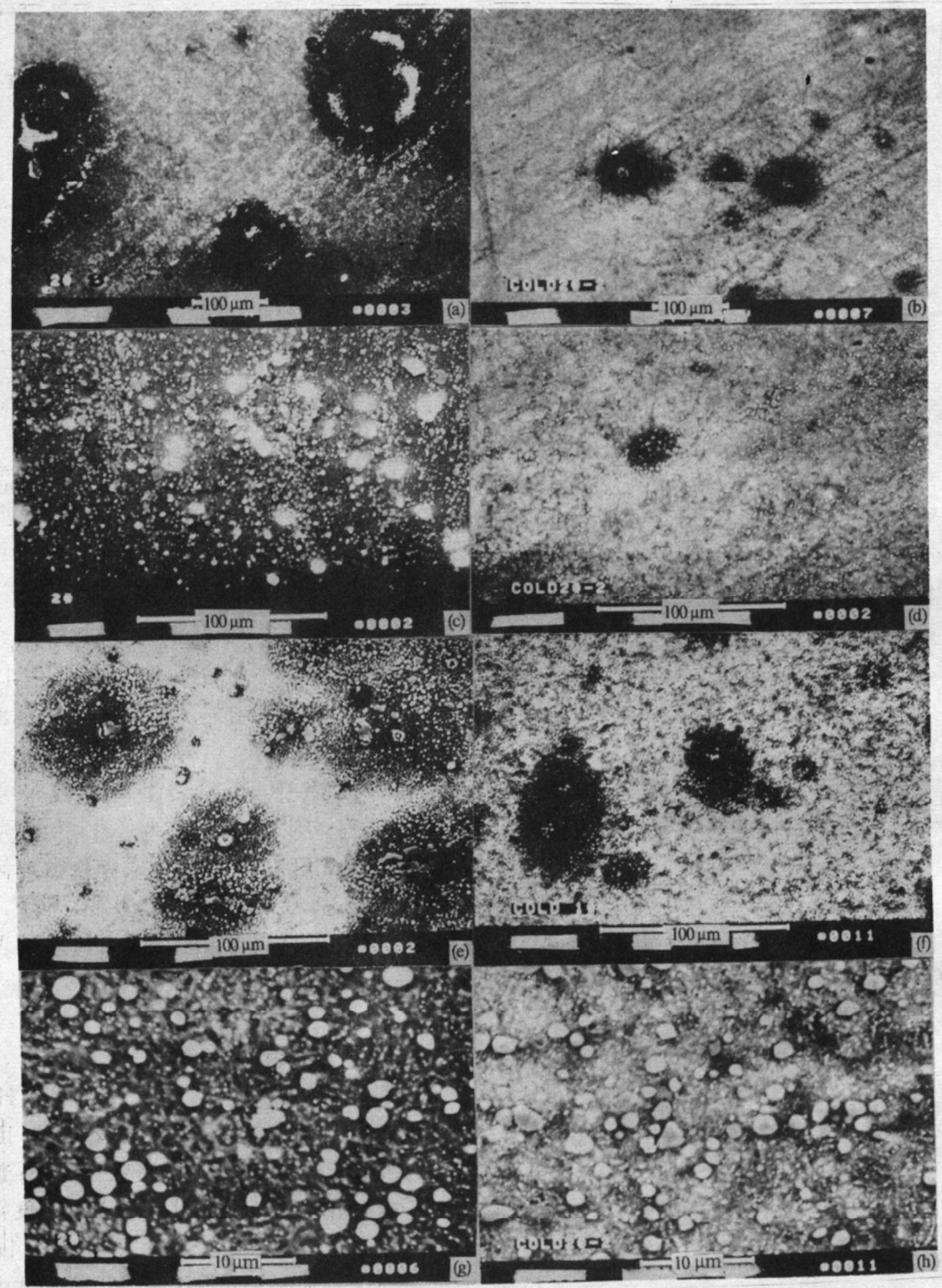

图 2 深冷处理前后微观组织的比较

(a), (c), (e), (g) 深冷处理前 SEM 照片; (b), (d), (f), (h) 深冷处理后 SEM 照片 
体基体. 表 2 中也列出了进行较仔细的选区测量后的测试结果.

深冷处理后显微硬度的变化是马氏体基体中含 $\mathrm{C}$ 量减少、细小碳化物的析出和大块碳化 物碎化等因素引起的.

\section{3 扫描电镜观察}

图 2 给出了一组扫描电镜照片, 直观地显示出深冷处理前后样品微观组织的明显差别. 从 200 倍和 500 倍的照片上都可以清楚看到: 处理前碳化物分布不够均匀, 且有较多大块碳化 物集中区. 深冷处理后, 在同样的放大倍数下看到碳化物的粒度明显减小, 分布均匀弥散, 大 块碳化物几乎看不到了. 产生这一过程有两种因素. 首先是深冷处理后许多大块碳化物碎化 为小块. 这从 (e), (f) 一对照片中可以看到, 处理前在衬度较深区域有大块碳化物聚集, 而在 处理后变成小块碳化物分布. 另一种因素是深冷处理后大量细小弥散的碳化物从马氏体晶粒 内部析出. 这一结论从 3000 倍的一对照片 (g), (h) 可以看得更为清楚. 处理前图 2(g) 还可以 看到由 VC 细小颗粒围成的一个个晶粒, 晶粒内部碳化物相对较少. 而在深冷处理后的照片 (h) 上, 看到晶粒内部又有很多非常细小的碳化物析出, 粒度估计是在 $0.1 \mu \mathrm{m}$ 以下. 这一实验 结果在文献[6] 中也有类似的报道.

用苦味酸酒精浸蚀剂, 可以使马氏体着色, 呈淡黄到棕色色调 (取决于金属中碳的含量), 这在 SEM 照片上表现为不同的衬度. 深冷处理前这种染色很不均匀, 这在 200 倍和 500 倍的照片 上都能看到. 着色不均匀反映出处理前样品内部马氏体的含量也是不均匀的. 棕色区含碳量 较高, 析出的碳化物相对较少, 且不够均匀和弥散. 而深冷处理后的样品, 利用同样的浸蚀剂和浸蚀 条件, 发现棕色区数量和面积明显减少, 说明深冷处理通过碳化物的析出使各区域马氏体的含 碳量趋于一致. 新析出的碳化物弥散细小, 加上大块碳化物的进一步碎化大大改善了.高速工 具钢中碳化物的分布状态.

\section{4 二次离子质谱测量}

利用二次离子质谱仪 (SIMS) 对深冷处理与未处理样品中碳的分布状况及含量变化情况 进行了对比分析, 分析样品是由 $\mathrm{W}_{18} \mathrm{Cr}_{4} \mathrm{~V}$ 高速钢刀具上截取下来的. 测试结果示于图 3 中. 我们看到, 处理后的样品中碳离子的分布区域明显地细微化了, 即深冷处理使原来同一碳分 布区域分解成若干块碳分布更为集中的小区, 而基体部分的碳含量相应减少. 这一测试结果 与上述的 X 射线衍射、显微硬度和扫描电镜的测试结果完全相符.

通过以上几种测试手段对深冷处理前后样品的综 合分析, 我们不难看到: 经淬火和回火处理后的高速工 具钢成品刀具, 其微观组织是马氏体基体中密布大量 的碳化物, 残余奥氏体含量非常少. 但在深冷处理 前, 碳化物的分布仍然是不够均匀的, 而且有一些大块 碳化物聚集区. 过去的研究工作 ${ }^{[4]}$ 已经表明, 碳化物 的分布状态, 颗粒的大小、形状, 大块碳化物的堆集等 都对高速钢的韧性、耐磨性和使用寿命有严重的影 响. 深冷处理致使马氏体, 特别是含碳量较高的区域 再度析出大量的细小又弥散的 $\mathrm{M}_{6} \mathrm{C}$ 型碳化物颗粒, 并 使大块碳化物碎化为小颗粒, 这种碳化物弥散而又均

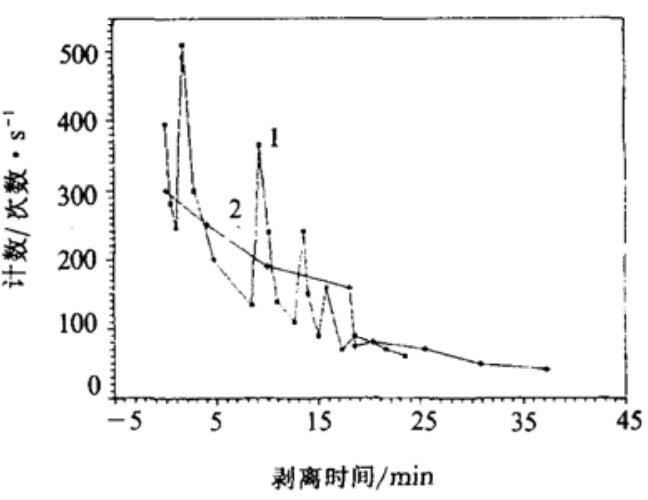

图 3 碳随剥离时间的分布曲线 1 - 经低温处理, 2 - 未经处理 
匀分布的微观组织是高速工具钢耐磨性和使用寿命提高 1 3 倍的主要原因.

\section{3 结论}

(1) 高速工具钢刀具经深冷处理后, 碳化物的粒度和分布的均匀性都有明显的变化. 这种 变化是稳定的, 不可逆的, 而且是从刀具表面到内部整体上的变化, 比一般表面强化有很大的 优越性. 这是深冷处理后刀具耐磨性和使用寿命提高 $1 \sim 3$ 倍的主要原因.

(2) 深冷处理后样品显微硬度略有降低, 这与马氏体中含碳量减少和碳化物变得更加细小 弥散有关. 因此刀具强化不是由于硬度提高所致.

(3) 回火处理后的成品刀具, 只含有极少量残余奥氏体, 所以残余奥氏体向马氏体的转化 也不是刀具使用寿命成倍提高的主要原因.

(4) 深冷处理工艺是有着重要应用价值的新技术, 低温强化机理的研究对进一步改善深冷 处理规程,扩大应用范围有着重要意义.

\section{参 考 文 献}

1 Dreger D R. The promise of cryogenic processing. Machine Design, 1981, 53(2):73 78

2 大和久重雄. Cryogenic treatment (日). 热处理, 1981, 21(1): $44 \sim 47$

3 Kurdjumov G V. Martensite crystal lattice, mechanism of austenite-martensite transformation and behavior of carbon atoms in martensite. Metall Trans, 1976, 7A(7): 999

4 上海机械制造工艺研究所. 金相分析技术. 上海: 上海科学技术文献出版社, 1987. $377 \sim 410$

5 刘 宁, 邓宗钢, 程本培. $\mathrm{W}_{6} \mathrm{Mo}_{5} \mathrm{Cr}_{4} \mathrm{~V}_{2}$ 高速钢激光相变硬化机理. 科学通报, 1993, 38(14): 1336

6 李智超,杨月君, 王洪才. 液氮低温处理对金属材料机械性能的影响. 低温超导, 1987, 15(4): 1 7 